\title{
Viscoelastic modeling of the fusion of multicellular tumor spheroids in growth phase
}

\author{
Guillaume Dechristé*1, Jérôme Fehrenbach ${ }^{\dagger 2}$, Elena Griseti ${ }^{\ddagger 2}$, Valérie \\ Lobjois $^{\S 2}$, and Clair Poignard ${ }^{\uparrow 1}$ \\ ${ }^{1}$ Team MONC, INRIA Bordeaux-Sud-Ouest, CNRS UMR 5251, 351 cours \\ de la Libération, 33405 Talence Cedex, France \\ ${ }^{2}$ Université de Toulouse, ITAV, CNRS, Toulouse, France
}

May 3, 2018

\begin{abstract}
Background. Since several decades, the experiments have highlighted the analogy of fusing cell aggregates with liquid droplets. The physical macroscopic models have been derived under incompressible assumptions. The aim of this paper is to provide a $3 \mathrm{D}$ model of growing spheroids, which is more relevant regarding embryo cell aggregates or tumor cell spheroids.

Methods. We extend the past approach to a compressible 3D framework in order to account for the tumor spheroid growth. We exhibit the crucial importance of the effective surface tension, and of the inner pressure of the spheroid to describe precisely the fusion. The experimental data were obtained on spheroids of colon carcinoma human cells (HCT116 cell line). After 3 or 6 days of culture, two identical spheroids were transferred in one well and their fusion was monitored by live videomicroscopy acquisition each 2 hours during $72 \mathrm{~h}$. From these images the neck radius and the diameter of the assembly of the fusing spheroids are extracted.

Results. The numerical model is fitted with the experiments. It is worth noting that the time evolution of both neck radius and spheroid diameter are quantitatively obtained. The interesting feature lies in the fact that such measurements characterise the macroscopic rheological properties of the tumor spheroids.
\end{abstract}

*guillaume.dechriste@gmail.com

†jerome.fehrenbach@math.univ-toulouse.fr

†elena.griseti@ipbs.fr

$\S$ Valerie.lobjois@itav.fr

ฯ Corresponding author, clair.poignard@inria.fr 
Conclusions. The experimental determination of the kinetics of neck radius and overall diameter during spheroids fusion characterises the rheological properties of the spheroids. The consistency of the model is shown by fitting the model with two different experiments, enhancing the importance of both surface tension and cell proliferation.

General Significance. The paper sheds new light on the macroscopic rheological properties of tumor spheroids. It emphasizes the role of the surface tension and the inner pressure in the fusion of growing spheroid. Under geometrical assumptions, the model reduces to a 2-parameter differential equation fit with experimental measurements. The 3-D partial differential system makes it possible to study the fusion of spheroids in non-symmetrical or more general frameworks.

Keywords: Tumor spheroid modeling;Surface Tension; Stokes descriptions; Fusing cell Aggregates

\section{Introduction}

\subsection{Motivations}

For a decade, the fusion of cell aggregates has become a booming subject in bioengineering science. Biological self-assembly is the cornerstone of bioprinting techniques for tissue reconstruction $[24,25,10,30]$, which provide an alternative to classic solid scaffold-based approaches in tissue engineering. In cancer biology, tumor spheroids provide interesting in vitro meso-scale tools, which can help in a better understanding of the tumor organisation and of its viscoelastic properties, at least at the early stage of the tumor development [21]. Indeed, tumor spheroids are interesting tools to reveal the macroscopic rheological properties of tumors. It is worth noting that such physical properties are crucial to accurately describe the tumor growth. Fusion of cell aggregates is also found in several development mechanisms, such as in early heart formation [33, 43]. In this respect, cellular aggregates are commonly modeled as viscoelastic fluids. Actually, their fusion is highly reminiscent of the coalescence of liquid drops, driven by an effective surface tension, by analogy with the surface tension in liquid drops. Furthermore, other experiments exhibit cellular tendency to minimize interfacial area, which can be interpreted as a consequence of surface tension. For instance, a mixture consisting of two different kinds of cell tends to form an aggregate where the two cell populations are sorted, as presented in Sun and Wang [40].

The analogy between the fusion of soft tissues and droplet liquids has been observed for several decades. One can cite for instance the cell sorting experiments on the early embryonic tissue behavior [38] or more recently the evolution of aggregate from irregular to spherical shape [31]. Moreover, specific devices have been developed to measure the viscoelastic properties of cell assembly $[12,14,13]$. Regarding the fusion of cell assemblies, the experimental data consist in measuring the so-called neck diameter of the fusion, which is the length of the line contact between the 2 spheroids on a planar projection (it is twice the radius neck $r$ of Figure 1 ). 
As far as we know, the previous studies have been derived in an incompressible framework. In this paper we are interested in modeling the fusion of multicellular tumor spheroids, that are specific spheroids which grow during the experiments. Such a growth, which violates the incompressibility assumption, cannot be neglected to account precisely for the fusion. Experimental measurements of the diameter of the spheroid assembly shows the crucial importance of this growth: under volume conservation assumption, the increase of the neck radius implies necessarily the decrease of the diameter of the spheroids in fusion, while the experiments exhibit an increase or at least a stabilization of this diameter. We aim at providing a new viscoelastic modeling of the fusion of such growing cell aggregates, performing an analogy with droplet fusion, as proposed since several decades, but getting rid of the incompressible condition.

Growth and velocity field are tightly linked since the divergence of the velocity is the local growth rate of the tumor. In standard models of tumor growth, as proposed by Greenspan [19], the choices of closure to determine the velocity are somehow arbitrary. The simplest choice consists of the standard Darcy's law, but Stokes equation or more complex rheological laws have been studied $[4,35]$. In this paper, we show that stuyding the fusion of tumor spheroids makes it possible to choose the viscoelastic law which is relevant with the experiments. We show that Stokes equation with a surface tension enables to recover the experimental observations. This approach exhibits the crucial role of surface tension and of the inner pressure on the growth and the fusion of spheroids. Our approach extends to the compressible framework the previous studies of cell aggregates fusion. An interesting feature of our approach lies in the fact that from experimental measurements, one can discriminate the rheological (viscoelastic) properties of the spheroid assembly from its growth parameters, which highly depend on the nutrient supply and the experimenal set-up. In particular, we show that under simple geometrical symmetry, the measurements of the neck radius and the diameter of the fusing spheroids assembly determine entirely the growth of the fusing spheroids. In addition, the macroscopic rheological parameters of the spheroid, which consist of the visco-capillary velocity and the pressure-viscosity ratio are entirely characterized by these measurements.

Remark 1 Throughout the paper, we refer to the effective surface tension of spheroids, by analogy with the surface tension in fluid mechanics. However it is worth noting that multicellular spheroids are much more complex than liquid drops. The observed surface tension results probably from more complex biological phenomena, which are still unclear.

\subsection{The standard modeling of spheroids fusion}

Theoretical models for the coalescence of two identical spherical droplets of high viscous fluid under the action of surface tension have been proposed within the framework of sintering $[15,32]$. In these papers, two spheres of radius $a$ and center $\mathrm{A}$ and $\mathrm{B}$ respectively that have one contact point $\mathrm{O}$ are considered (see Figure 1). During the fusion, both centers move towards the point $\mathrm{O}$ and the angle $\theta=\widehat{\mathrm{OBI}}$, where $\mathrm{I}$ is an intersection point between the spheres, goes from 0 to $\frac{\pi}{2}$, as reported in Figure 1 . 


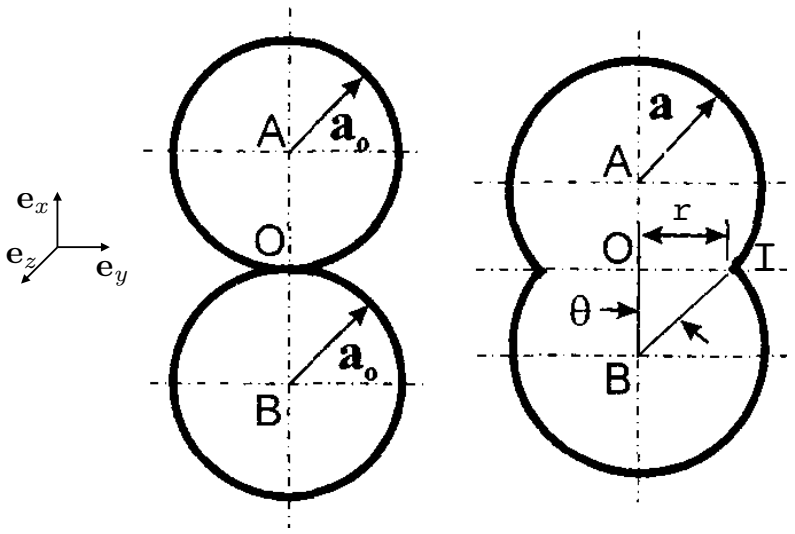

Figure 1: Schematic descriptions of the fusion as defined by Shaler, Frenkel et al [15] and Pokluda et al. [32].

Equaling the works of the effective surface tension and of the viscous dissipation, the authors found a relation between the angle $\theta$, the radius $a$ and their time derivatives. Adding the volume conservation hypothesis leads to the following ordinary equation on $\theta$ as presented by Pokluda et al. - see equation (14), pp 3254 [32]:

$$
\dot{\theta}=\frac{1}{2} \frac{\Gamma}{\eta a_{0} \theta}
$$

where $\eta$ is the viscosity of the spheroid, $\Gamma$ is the effective surface tension and $a_{0}$ the initial radius of the spheroid, before the fusion. The ratio $\Gamma / \eta$ is referred to as the viscocapillary velocity. Several studies have adapted this approach in its simplified version to model the radius neck during the fusion of spheroids $[11,23,10]$ by setting

$$
r^{2}=2^{\frac{2}{3}} a_{0}^{2}\left(1-e^{-t / \tau}\right),
$$

where $\tau=2^{\frac{2}{3}} a_{0} \eta / \Gamma$. The above approach has been barely validated in the case where the fusion is much faster than the cell division. However, regarding growing spheroids, and in particular tumor spheroids, the time scale of fusion is much larger than the time of cell division. Moreover the exponential term has been set to fit with the experiments but it is not justified by physical considerations. For all these reasons, it seems necessary to provide a new model for the fusion of growing spheroids.

In addition, in this paper we are interested in studying the fusion of tumor spheroids, during several tens of hours (up to 72 hours). Such cell spheroids are proliferating and the volume conservation is not satisfied: the previous approach of Shaler, Frenkel et al. fails to provide relevant results. One of the most clear justification of such inconsistency is provided by the measurement of the spheroid diameter (the largest diameter of the spheroid), which stays constant or even increases during the experiments, while the theory of Shaler, Frenkel et al predicts a decreasing. 


\subsection{Goal and outline of the paper}

Among the mathematical models used to describe the fusion of cell aggregates and to optimize biofabrication, Monte Carlo methods on the cell-to-cell interactions have been widely used $[16,40,41]$. In this microscopic approach, each cell is discretized and its motion results from its interactions with its neighbors. Agent-based models makes it possible to include many biological phenomena to study the spheroids behaviors under different experimental configurations. In particular, one can cite the multiphysics software CellSys [22], which enables to incorporate many physical behaviours of cell spheroids. Such models are well-suited for the study of all the mechanisms acting at the cellular level, such as cell adhesion and motility. However due to their computational cost, such methods are not suited to deal with large spheroids or tumors with more than several tens of thousands of cells.Morever, beyond their computational costs it is still a current challenge to link these cell-scale models with macroscopic viscoelastic properties. Our goal is different in the sense that we aim to study the viscoelastic properties of spheroids to infer the rheological properties of tumors.

Indeed, we are generally interested in macroscopic tumor growth models, with macroscopic viscoelastic properties. For such a purpose, we derive a continuum mechanics model of the fusion of cell spheroids. The model is based on the analogy of cell spheroid with viscous fluid with specific the surface tension. The growth of the spheroid is accounted for by a standard growth law model, which consists in a simple ordinary differential equation proposed by Gompertz [18] for instance. Other more complex models involving hyperbolic partial differential equations can also be chosen, as discussed in Michel's PhD thesis [29]. The growth model leads to the divergence of the velocity field inside the spheroid, which is governed by Stokes equation. The analogy with liquids is modeled thanks to the introduction of an effective surface tension. Interestingly, we show how the approaches of Shaler, Frenkel et al. and later on the works of Pokluda et al. are simplifications of our model, under the incompressibility hypothesis. Our approach is validated by quantitative comparisons of the numerical and experimental measurements of the neck radius and the spheroid diameter.

The outline of the paper is as follows. In the next section, we provide the complete partial differential system based on Stokes equation, describing the fusion of growing spheroid. The complete model combines a tumor growth model with a viscous model describing the velocity field. Interestingly, both model are linked thanks to the divergence of the velocity field, which equals the rate of volume changes. Under symmetry assumptions similar to Pokluda et al., we simplify the 3D model into a 0D model which consists in an ordinary differential system. We show that under the incompressibility assumption, our 0D model is similar to the model of Pokluda et al., but it is formulated in a simple way, that prevents numerical issues unlike the model of Pokluda et al. We conclude by comparing the 0D model with experimental data, proving the consistency of the modeling. 


\section{Viscoelastic model of the fusion of growing spheroids}

The purpose of this section is to propose a model, which accounts simultaneously for the spheroids growth and their fusion. The spheroids are seen as viscous fluid with specific rheological properties. To account for the tumor growth, the cell densities are generically supposed to be driven at a velocity whose divergence equals the production terms, as proposed by Greenspan [19] in the late 70's, Byrne et al. [6] and more recently by Colin, Saut et al. $[5,2,7]$. Then a phenomenological law such Darcy or Stokes law is used to close the system. Such a modeling involves parameters, whose determination needs advanced experimental techniques. For instance determining the proportion of proliferating and quiescent cells can be performed thanks to metabolic rate activity measurements or by examining specific markers such as BrdU or EdU. Oxygen and nutrient consumption rates are also a crucial in tumor proliferation $[17,28]$. These models are interesting to investigate the influence of the different configurations on the tumor growth, but given a specific experimental set-up, it is hard to determine which parameters have to be used a priori. Regarding the fusion of spheroids, which is the goal of the paper, we interesting show that measuring the neck radius and the spheroids assembly diameter gives the growth curve of the spheroid. Therefore the growth law is no more an unknown but it is given by the experiments. The remaining unknowns are the macroscopic rheological properties, which are somehow intrinsic to the spheroid cell type, and can be fitted by the experiments. It is worth noting that these rheological parameters can be hardly measured, but they can be obtained by fitting the model with the experimental measurements. Even though such full 3D models are the

most relevant to describe in general the fusion of spheroids, it is interesting to simplified the tumor growth model into a 0D model for calibration purpose. Such a 0D simplification is based on the following assumptions:

- The growth of the spheroid volume can be described by an simple ordinary equation.

- The 2 spheroids in fusion are identical.

\subsection{Growth law and area and volume of the fusing spheroids}

Denoting by $f$ the volume of one spheroid, let assume that $f$ follows the following ordinary differential equation:

$$
\dot{f}(t)=\varphi(f), \quad f(0)=f_{0} .
$$

This class of ordinary differential equation for tumor growth has been used in preclinical studies for several decades, essentially to track the tumor volume dynamics in mice models. One can cite the papers of Von Bertalanffy [42], Laird [27], Spratt et al. [37], or more recently Wilson et al. [44] for instance. We refer to Benzekry et al. [1] for an exhaustive comparison of the many different 0D-tumor growth models that can be found in the literature. The choice of the function $\varphi$ is related to the experimental observations on the growth of single spheroid. For instance, a power law can be used - that is $\varphi(f)=\alpha f^{\beta}$ - but Gompertz 
model is widely used - that is $\varphi(f)=\beta f \log (\alpha / f)$, where $\alpha$ and $\beta$ are the parameters of the growth, and $f_{0}$ is the volume of the spheroid at the beginning of the sintering, when the two spheroids have only 1 contact point.

Denoting by $\mathcal{A}$ and $\mathcal{V}$ the area and the volume of the assembly of the spheroids in fusion, simple geometric considerations based on Figure 1 imply that

$$
\begin{aligned}
& \mathcal{A}(t)=4 \pi a^{2}(t)(1+\cos (\theta(t))), \\
& \mathcal{V}(t)=\frac{2 \pi}{3} a^{3}(t)\left(2+3 \cos (\theta(t))-\cos (\theta(t))^{3}\right)=2 f(t),
\end{aligned}
$$

where $a$ is the radius of one of the spheroid in fusion, and $\theta$ is the acute angle between the axis (OA) and the line (AI) (see Figure 1). The neck radius $r$ is just given by

$$
r(t)=a(t) \sin (\theta(t))
$$

and the spheroid diameter reads

$$
d(t)=2(a(t)+a(t) \cos (\theta(t)))
$$

\subsection{Stokes equation}

The spheroids are seen as viscous liquid with a viscosity $\eta$ and a surface tension $\Gamma$. The velocity field $\mathbf{v}$ and the pressure $\Pi$ are unknowns which satisfy the so-called Stokes equation

$$
\nabla \cdot \overline{\bar{T}}=0, \quad \text { in } \mathcal{S}(t), \quad \text { with } \quad \overline{\bar{T}}=\left[2 \eta \underline{D}[\mathbf{v}]-\left(\frac{2}{3} \eta \nabla \cdot \mathbf{v}+\Pi\right) \underline{\text { Id }}\right]
$$

where $\underline{D}[\mathbf{v}]=\frac{1}{2}\left(\nabla \mathbf{v}+\nabla \mathbf{v}^{T}\right)$ is the rate of deformation tensor. Assuming that the spheroid is homogeneous, thanks to the so-called Reynolds formula ${ }^{1}$ the divergence of $\mathbf{v}$ is given by

$$
\nabla \cdot \mathbf{v}=\frac{\dot{\mathcal{V}}}{\mathcal{V}}=\frac{\varphi(f)}{f}, \quad \text { in } \mathcal{S}(t)
$$

On the boundary of the spheroid, the surface tension minimizes the mean curvature $\kappa=\nabla \cdot \mathbf{n}$ :

$$
\overline{\bar{T}} \mathbf{n}=2 \eta \underline{D}[\mathbf{v}] \mathbf{n}-\left(\frac{2}{3} \eta \nabla \cdot \mathbf{v}+\Pi\right) \mathbf{n}=\Gamma \kappa \mathbf{n}, \quad \text { on } \partial \mathcal{S}(t) .
$$

The boundary of the fusing spheroids is then transported by the normal velocity $\mathbf{v}$.

\subsection{Ordinary Differential systems for the fusion of growing spheroids}

The above problem can be simplified into a 0D differential system. More precisely, one can recover the approach of Shaler, Frenkel et al. [36], and more recently Pokluda et al. 
Multiplying the Stokes equation by $\mathbf{v}$ and integrating by part, one obtains the following equality:

$$
\int_{\mathcal{S}(t)} \overline{\bar{T}}: \nabla \mathbf{v} d x=\int_{\partial \mathcal{S}(t)} \Gamma \kappa \mathbf{v} \cdot \mathbf{n} d s
$$

This is exactly the balance between the work $\dot{\mathcal{W}}_{v}$ of the viscosity forces per unit of time, and the work per unit of time of the effective surface tension $\dot{\mathcal{W}}_{s}$ as stated by Shaler, Frenkel, Pokluda et al. The difficulty here lies in the fact that the spheroids are growing, and thus the assembly has to be seen as a compressible fluid, whose divergence is linked to the rate of change of the volume. The right hand side of (8) is nothing but the change of the area multiplied by the surface tension as shown by Grinfeld [20]:

$$
\int_{\partial \mathcal{S}(t)} \Gamma \kappa \mathbf{v} \cdot \mathbf{n} d s=-\Gamma \frac{d \mathcal{A}}{d t} .
$$

Assuming that the stress tensor and the gradient of the velocity are constant in the cell aggregate, the work of viscous forces per unit of time $\dot{\mathcal{W}}_{v}$-that is the left hand side of (8)at any point of the spheroid is similar to the same work per unit of time at the point A (see Figure 1):

$$
\dot{\mathcal{W}}_{v}=\mathcal{V}(t)\left\{\eta\left(\nabla \mathbf{v}(\mathrm{A})+\nabla \mathbf{v}^{\mathrm{T}}(\mathrm{A})\right): \nabla \mathbf{v}(\mathrm{A})-\frac{2}{3} \eta\left(\frac{\varphi(f)}{f}\right)^{2}-\Pi \frac{\varphi(f)}{f}\right\} .
$$

Using the symmetry one infers that the $\partial_{x} \mathbf{v}_{x}(\mathrm{~A})=\partial_{z} \mathbf{v}_{z}(\mathrm{~A})$, where the $x-, y-$, and $z-$ directions are given in Figure 1. In addition, since the displacement is only along the axis $\mathbf{e}_{y}$, one also has

$$
\partial_{z} \mathbf{v}_{x}(\mathrm{~A})=\partial_{\mathrm{x}} \mathbf{v}_{\mathrm{z}}(\mathrm{A})=\partial_{\mathrm{z}} \mathbf{v}_{\mathrm{y}}(\mathrm{A})=\partial_{\mathrm{y}} \mathbf{v}_{\mathrm{z}}(\mathrm{A})=\partial_{\mathrm{x}} \mathbf{v}_{\mathrm{y}}(\mathrm{A})=\partial_{\mathrm{y}} \mathbf{v}_{\mathrm{x}}(\mathrm{A})=0 .
$$

Thanks to the divergence condition (6), one infers

$$
2 \partial_{x} \mathbf{v}_{x}(\mathrm{~A})+\partial_{\mathrm{y}} \mathbf{v}_{\mathrm{y}}(\mathrm{A})=2 \partial_{\mathrm{z}} \mathbf{v}_{\mathrm{z}}(\mathrm{A})+\partial_{\mathrm{y}} \mathbf{v}_{\mathrm{y}}(\mathrm{A})=\frac{\varphi(\mathrm{f})}{\mathrm{f}},
$$

and therefore

$$
\left(\nabla \mathbf{v}(\mathrm{A})+\nabla \mathbf{v}^{\mathrm{T}}(\mathrm{A})\right): \nabla \mathbf{v}(\mathrm{A})=2\left(\partial_{y} \mathbf{v}_{y}(\mathrm{~A})\right)^{2}+\left(-\partial_{\mathrm{y}} \mathbf{v}_{\mathrm{y}}(\mathrm{A})+\frac{\varphi(\mathrm{f})}{\mathrm{f}}\right)^{2}
$$

Similarly to Pokluda et al., approximating $\partial_{y} \mathbf{v}_{y} \sim \frac{1}{a \cos \theta} \frac{d}{d t}(a \cos \theta)$ one infers ${ }^{2}$

$$
\left(\nabla \mathbf{v}(\mathrm{A})+\nabla \mathbf{v}^{\mathrm{T}}(\mathrm{A})\right): \nabla \mathbf{v}(\mathrm{A})=3\left(\frac{\dot{w}}{w}\right)^{2}-2 \frac{\varphi(f)}{f} \frac{\dot{w}}{w}+\left(\frac{\varphi(f)}{f}\right)^{2}
$$

\footnotetext{
${ }^{2}$ Let us mention that the formula (6) page 3254 should involve the term $\cos \theta$ at the denominator. This has no influence in Pokluda et al.'s paper since they consider small angle $\theta$ and thus $\cos \theta \sim 1$.
} 
where for conciseness $w=a \cos \theta$. Therefore, the work due to viscous forces per unit of time is given by

$$
\dot{\mathcal{W}}_{v}=2 f(t)\left\{3 \eta\left(\frac{\dot{w}}{w}\right)^{2}-2 \eta \frac{\varphi(f)}{f} \frac{\dot{w}}{w}+\frac{1}{3} \eta\left(\frac{\varphi(f)}{f}\right)^{2}-\Pi \frac{\varphi(f)}{f}\right\} .
$$

On the other hand, the work $\dot{\mathcal{W}}_{s}$ due to the surface tension per unit of time is given by

$$
\dot{\mathcal{W}}_{s}=-\Gamma \frac{d \mathcal{A}}{d t}=-4 \pi \Gamma(\dot{a}(2 a+w)+a \dot{w}) \text {. }
$$

Therefore, identifying the works per unit of time due to the viscous forces and to the surface tension, and using the volume formula (4) one infers the nonlinear differential system satisfied by $a$ and $w$ :

$$
\begin{aligned}
& 2 f(t)\left\{3 \eta\left(\frac{\dot{w}}{w}\right)^{2}-2 \eta \frac{\varphi(f)}{f} \frac{\dot{w}}{w}\right. \\
& \left.+(\eta+\xi)\left(\frac{\varphi(f)}{f}\right)^{2}-\Pi \frac{\varphi(f)}{f}\right\}=-4 \pi \Gamma(\dot{a}(2 a+w)+a \dot{w}), \\
& 2 a^{3}+3 a^{2} w-w^{3}=\frac{3}{\pi} f
\end{aligned}
$$

where $f$ is entirely determined as the solution to (2). Deriving (11b), one gets

$$
\dot{a}=\frac{1}{2 \pi} \frac{\varphi(f)}{a(a+w)}+\frac{w-a}{2 a} \dot{w}
$$

and then one infers

$$
\begin{aligned}
& \left(\frac{\dot{w}}{w}\right)^{2}+\frac{2}{3}\left\{\pi \frac{\Gamma}{\eta} \frac{(w+a)}{f} \frac{w^{2}}{2 a}-\frac{\varphi(f)}{f}\right\} \frac{\dot{w}}{w}+\frac{1}{9}\left(\frac{\varphi(f)}{f}\right)^{2} \\
& -\frac{\Pi}{3 \eta} \frac{\varphi(f)}{f}+\frac{\Gamma}{3 \eta} \frac{\varphi(f)}{f} \frac{2 a+w}{a(a+w)}=0 .
\end{aligned}
$$

Defining the functions $(w, a, f) \mapsto B(w, a, f)$ and $(w, a, f) \mapsto C(w, a, f)$ by

$$
\begin{aligned}
& B(w, a, f)=\frac{w}{3}\left\{\pi \frac{\Gamma}{\eta} \frac{(w+a)}{f} \frac{w^{2}}{2 a}-\frac{\varphi(f)}{f}\right\} \\
& C(w, a, f)=\frac{\varphi(f)}{f} w^{2}\left(\frac{\Gamma}{3 \eta} \frac{2 a+w}{a(a+w)}+\frac{1}{9} \frac{\varphi(f)}{f}-\frac{1}{3 \eta} \Pi\right)
\end{aligned}
$$

we thus infer that the triplet $(f, w, a)$ is solution to the differential system

$$
\begin{aligned}
& \dot{f}=\varphi(f), \\
& \dot{w}=-B(w, a, f)-\sqrt{B(w, a, f)^{2}-C(w, a, f)}, \\
& \dot{a}=\frac{1}{2 \pi} \frac{\varphi(f)}{a(a+w)}+\frac{w-a}{2 a}\left(-B(w, a, f)-\sqrt{B(w, a, f)^{2}-C(w, a, f)}\right),
\end{aligned}
$$


with the initial conditions

$$
f(0)=f_{0}, \quad w(0)=w_{0}, \quad a(0)=a_{0},
$$

where $f_{0}, w_{0}$ and $a_{0}$ are the respective initial values of $f, w$ and $a$. Note that at the very begining of the sintering, $w=a$, however in the experiments the measurements start after this very begining, and the fusion has already started. The neck radius $r$ and the largest diameter $d$ of the assembly are then given by

$$
r(t)=\sqrt{a(t)^{2}-w(t)^{2}}, \quad d(t)=2(a(t)+w(t)) .
$$

Remark 2 (Fusion of non-growing spheroids) One can compare our approach to Pokluda et al. regarding the fusion of non growing spheroids. In this case, $\dot{f}=0$ and then the problem reads

$$
\begin{aligned}
& \dot{w}=-2 B\left(w, a, f_{0}\right), \\
& \dot{a}=-\frac{w-a}{a} B\left(w, a, f_{0}\right) .
\end{aligned}
$$

It is worth noting that this problem is well-posed for any initial conditions $a_{0}>0$ and $w_{0}>0$. Moreover, making the assumption of small angle $\theta$, and reminding that $w=a \cos \theta$, one infers that

$$
2 B\left(w, a, f_{0}\right) \sim \frac{\Gamma}{2 \eta} .
$$

Then, since $\cos \theta \sim 1-\theta^{2} / 2$, equation (15b) can be approached by

$$
\frac{a_{0}}{2} \frac{d\left(\theta^{2}\right)}{d t}=\frac{\Gamma}{2 \eta}
$$

which is exactly the differential equation of Pokluda et al. [32]:

$$
\dot{\theta}=\frac{\Gamma}{2 a_{0} \eta \theta}
$$

However, as noted by Pokluda et al., the later equation does not fall in the Cauchy-Lipschitz theory if $\left.\theta\right|_{t=0}=0$, while the exact system (17) remains well-posed with the initial conditions $w_{0}=a_{0}>0$, which correspond to $\theta=0, a(0)=a_{0}$.

Remark 3 (On the choice of the root of (12)) In equation (15b), the choice of the smallest root of (12) has been made. This choice is driven by the experimental data. To make thing clear, let us first consider the fusion of non growing spheroids. In this configuration, the term $C(w, a, f)$ vanishes and the two possible equations for $\dot{w}$ are:

$$
\dot{w}=0 \quad \text { or } \quad \dot{w}=-2 B(w, a, f) .
$$

Since fusion occurs in the experiments, necessarily the second equality has to be chosen. Actually the physiological values of the visco-capillary velocity in spheroids ranges in 1 to 
$0.1 \mu \mathrm{m} / \mathrm{min}$, while the pressure-viscosity ratio is about $10^{-3} \mathrm{~s}^{-1}$ in solid tumors and much lower in multicellular spheroids according to [39, 34, 3]. Moreover the experimental measurements show that the order of magnitude of $\dot{w}$ is about $1 \mathrm{~nm} / \mathrm{s}$, while $w$ and a are about several hundreds of microns. These rough estimates show that

$$
\sqrt{B(w, a, f)^{2}-C(w, a, f)} \sim B(w, a, f),
$$

and $\dot{w}$ is not negligible, this justifies choice of root of the polynomial in equation (15b).

\section{Calibration and comparison with experimental data}

This section is devoted to justifying the relevance of the modeling by comparison with experimental data.

\subsection{Experimental set-up}

Spheroids of colon carcinoma human cells (HCT116 cell line) were formed by using the centrifugation method in 96-well plates, as described by Desmaison et al. in [8]. Two HCT116 cell lines stably expressing either the H2B-mCherry red-emitting fluorescent fusion protein or the fusion protein H2B-GFP were used. After 3 or 6 days of culture, two spheroids at the same growth stage - one made of the HCT116-H2B-mCherry cells and the other one with the HCT116-H2B-GFP cells - were transferred in one well and their fusion was monitored by live videomicroscopy. In each experiment, the acquisition was performed for 10 wells in parallel, a z-stack with a $20 \mu \mathrm{m}$ z-step was acquired for each well to ensure obtaining in-focus images of the fusion zone. The total duration of the time lapse experiment was $72 \mathrm{~h}$ with an acquisition every 2 hours. Figure 2 provides an example of videomicroscopy images acquisition by the experimental set-up. From these images the neck radius and the diameter of the assembly of the fusing spheroids are extracted.

\subsection{Fusion of identical growing spheroids}

The calibration of the parameters involved in the $0 \mathrm{D}$ model is required to compare the numerical results with the experimental data. A priori, it is necessary to have the growth law of the spheroid, that is to have the expression of the function $\varphi(f) / f$ from $(2)$, as well as the ratio $\Gamma / \eta$ referred to as viscocapillary velocity [32] and the pressure-viscosity ratio $\Pi / \eta$. Therefore, considering for instance Gompertz growth law, one has a priori 4 parameters to fit.

The interesting point lies in the fact that taking advantage of the symmetry, as assumed for the 0D-model, one can obtain directly the growth law from the measurements of the

neck radius and of the spheroid diameter. More precisely, from (16), one also has the reverse maps from $(r, d)$ towards between $(a, w)$ : 


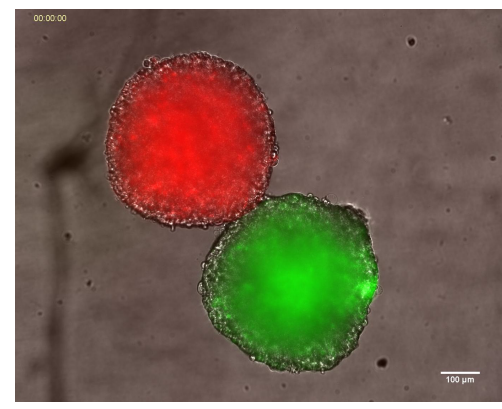

(a) Two spheroids at $t=0$.

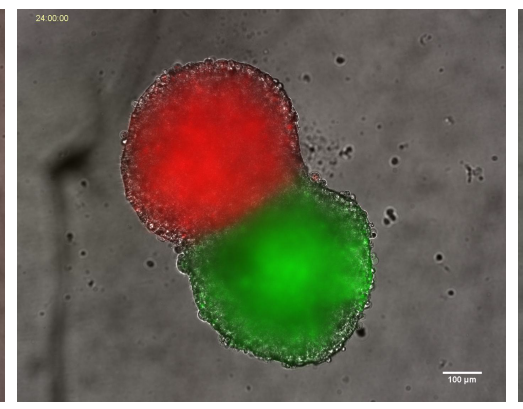

(b) Fusing spheroids at $t=24 h$.

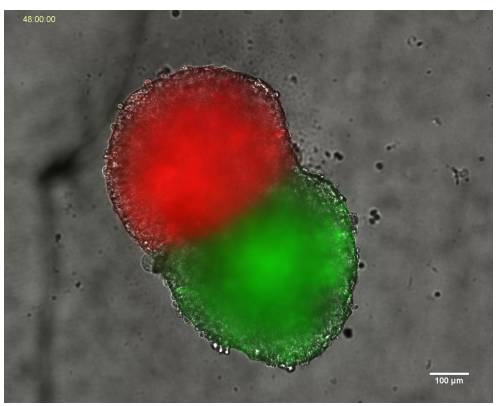

(c) Fusing spheroids at $t=48 \mathrm{~h}$.

Figure 2: Images of the fusing spheroids acquired from the live videomicroscopy at $t=0$ (Left), $t=24 h$ (Center) and $t=48 h$ (Right). An acquisition was perfomed every 2hours for a total duration of the experiments of $72 \mathrm{~h}$. Grey: transmitted-light, red: mCherry fluorescence, green: GFP fluorescence. Scale bar: $100 \mu \mathrm{m}$.

$$
a(t)=\frac{1}{4} d(t)+\frac{r^{2}(t)}{d(t)}, \quad w(t)=\frac{1}{4} d(t)-\frac{r^{2}(t)}{d(t)},
$$

and then the volume of the spheroids in fusion $\mathcal{V}$ is given by equation (11b). Therefore from the time-measurements of $r$ and $d$, one extracts the evolution of the spheroid volume, which then gives the function $\varphi(f) / f$.

Therefore the number of parameters is reduced to the viscocapillary velocity $\Gamma / \eta$ and the pressure-viscosity ratio $\Pi / \eta$. The data provided by Figure 3 justify straightforwardly the motivation of our approach since the volume of the spheroid assembly is far from constant.

Knowing the ratio $\varphi(f) / f$, the differential equations (15) are then solved with the routine odeint from the python library SciPy [26], which is optimized to solve ordinary differential systems. From then, we obtain the best fits of the data for both experiments with the value $\Gamma / \eta=3.10^{-3} \mu \mathrm{m} / \mathrm{s}$, and $\Pi / \eta=1.0 \mu \mathrm{s}$, which seems consistant with the value of viscocapillarity that can be found in the literature ${ }^{3}$. Figure 4 and Figure 5 show that the 0D model provides consistant results. It worth noting that the approach of Frenkel, et al. as used in $[9,39]$ would have failed since the diameter of the spheroid is far from decreasing, as reported by Figure 5 .

\section{Conclusion}

In this paper, we have presented a viscoelastic model of the fusion of growing spheroids which extends the classical approach used since 90's. Our model combines a classical tumor growth model as proposed by Greenspan [19] and used extensively by Colin, Saut et al. To close the

\footnotetext{
${ }^{3}$ Stirbat et al. found $7.6 \mu \mathrm{m} / \mathrm{s}$ for the visco-capillarity velocity of aggregate of mouse embryonic carcinoma F9 cell line [39].
} 


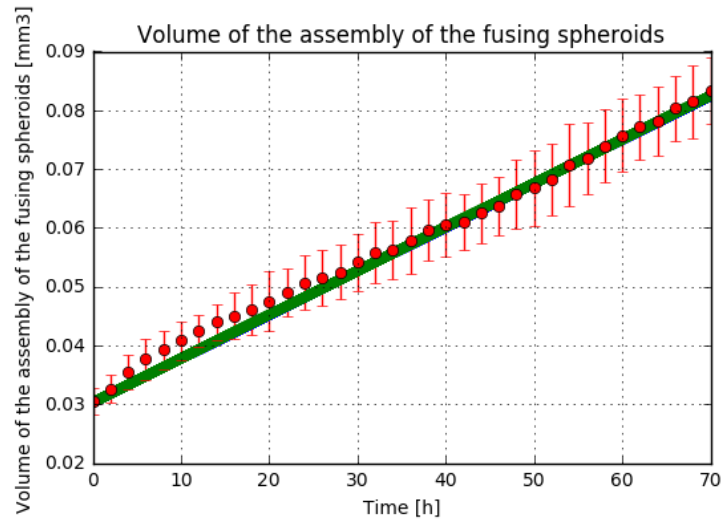

(a) Fusion of 2 spheroids of $300 \mu \mathrm{m}$ diameter.

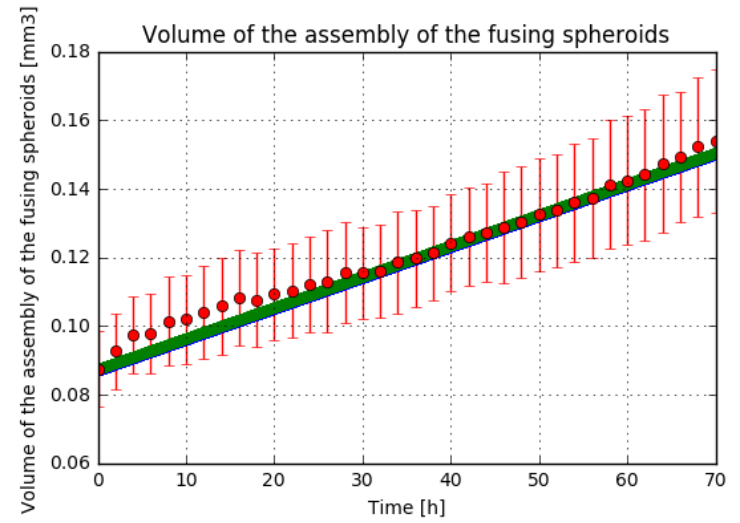

(b) Experiment with 2 spheroids of $500 \mu \mathrm{m}$ diameter.

Figure 3: Spheroid volume evolution obtain from the measurements of the neck radius and the spheroid diameter. For both experiments the evolution is almost linear. For each setup, 6 experiments have been performed.

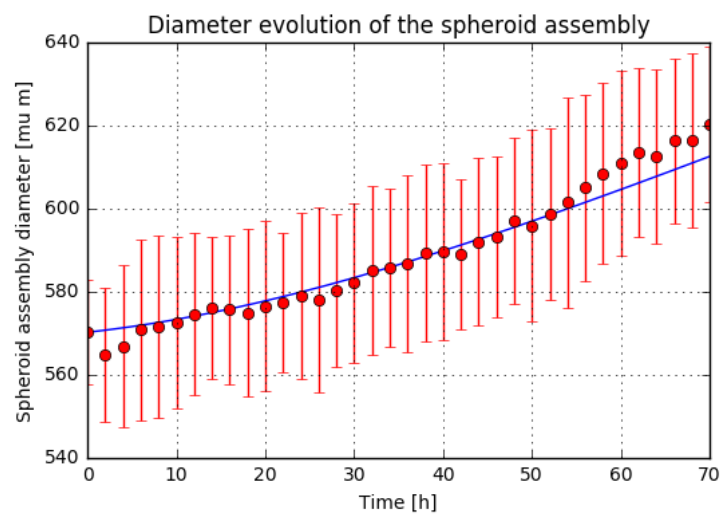

(a) Fusion of 2 spheroids of $300 \mu \mathrm{m}$ diameter.

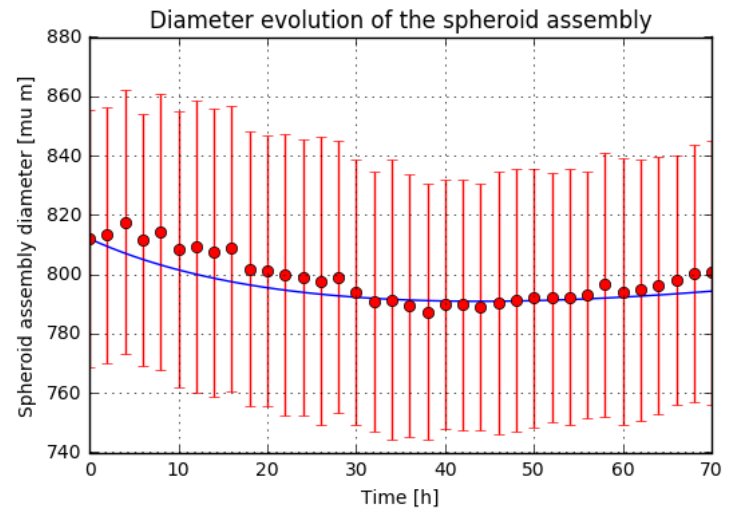

(b) Experiment with 2 spheroids of $500 \mu \mathrm{m}$ diameter.

Figure 4: Radius neck evolution obtained from the measurements (red dots) and from the model (continuous line), with the parameter values $\Gamma / \eta=3.10^{-3} \mu \mathrm{m} / \mathrm{s}$, and $\Pi / \eta=1.0 \mu \mathrm{s}$.

system, the simple Darcy law used in $[2,5]$ is replaced by Stokes equation for compressible fluid with an effective surface tension. This complex 3D model is simplified into an ordinary differential system of 3 unknowns, which is easy to solve with standard ODE solver. Using the Python library odeint, we obtain a set of parameters for which the numerical results are closed to the data. Interestingly, in the case of non growing spheroid, our approach is equivalent with the approach of Pokluda et al., but our system is well-posed for any positive initial data. It thus avoids the numerical difficulties mentioned in [32].

The biological outcome of our results lies in the fact that measuring the neck radius 


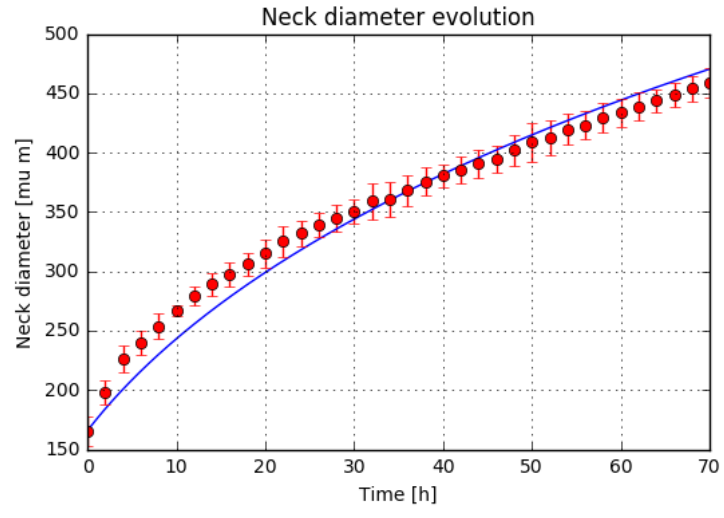

(a) Fusion of 2 spheroids of $300 \mu \mathrm{m}$ diameter.

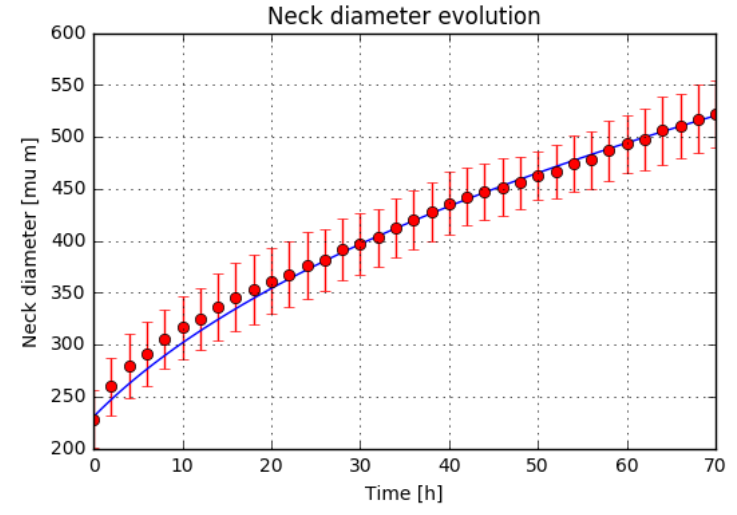

(b) Experiment with 2 spheroids of $500 \mu \mathrm{m}$ diameter.

Figure 5: Spheroid diameter evolution obtained from the measurements (red dots) and from the model (continuous line), with the parameter values $\Gamma / \eta=3.10^{-3} \mu \mathrm{m} / \mathrm{s}$, and $\Pi / \eta=1.0 \mu \mathrm{s}$.

and the diameter of the spheroid assembly makes it possible to characterize the macroscopic viscoelastic properties of the spheroids. The experimental data make it possible to obtain the visco-capillary velocity and the pressure-viscosity ratio, which are the parameters that describe the rheological properties of the spheroids. The way the viscocapillary velocity and the pressure-viscosity ratio behave in term of tumor aggressiveness is still unclear. A forthcoming study would compare the rheological properties of different tumor spheroids aggressiveness, in the sense of growth velocity.

Interestingly, from the continuum fluid mechanics, we recover and extended the 0D model previously obtained by Douezan et al. [9], and Pokluda et al. [32] and more recently Jakab et al. [25]. Since our model is written in the general 3D configuration, it makes it possible to study the fusion of spheroids in non-symmetrical configurations. Moreover, the study of multicellular spheroids, with different cell species is possible thanks to our approach. For these reasons, this paper provides a way to better understand the rheology of tumor at least at its early stage. It enhances the importance of the effective surface tension in the fusion of cell aggregates.

\section{Acknowledgements}

This work was supported by ITMO Cancer AVIESAN (Alliance Nationale pour les Sciences de la Vie et de la Santé, National Alliance for Life Sciences \& Health) within the framework of the Plan Cancer and la Ligue Contre le Cancer (comité de la Haute-Garonne). The authors wish to acknowledge the TRI-Genotoul and ITAV imaging facilities. We thank Bernard Ducommun and Thierry Colin for their helpful comments on the manuscript and the members of our groups for discussions and interest to this project.

C.P. is partly supported by the Plan Cancer projects DYNAMO (PC2015-15) and NU- 
MEP (PC2016-15). CP is grateful to the Dept. of Mechanical Engineering of UCSB for its hospitality, which made it possible to finalise the manuscript.

\section{References}

[1] S. Benzekry, C. Lamont, A. Beheshti, A. Tracz, J. M. L. Ebos, L. Hlatky, and P. Hahnfeldt. Classical mathematical models for description and prediction of experimental tumor growth. PLOS Computational Biology, 10(8):1-19, 082014.

[2] F. Billy, B. Ribba, O. Saut, H. Morre-Trouilhet, T. Colin, D. Bresch, J.-P. Boissel, E. Grenier, and J.-P. Flandrois. A pharmacologically based multiscale mathematical model of angiogenesis and its use in investigating the efficacy of a new cancer treatment strategy. Journal of theoretical biology, 260(4):545-562, 2009.

[3] A. Blumlein, N. Williams, and J. J. McManus. The mechanical properties of individual cell spheroids. Scientific Reports, 2017.

[4] D. Bresch, T. Colin, E. Grenier, B. Ribba, and O. Saut. A viscoelastic model for avascular tumor growth. Conference Publications, 2009(0133-0189-2009-Special-101), 2009.

[5] D. Bresch, T. Colin, E. Grenier, B. Ribba, and O. Saut. Computational modeling of solid tumor growth: The avascular stage. SIAM Journal on Scientific Computing, 32(4):2321-2344, 2010.

[6] H. Byrne, J. King, D. McElwain, and L. Preziosi. A two-phase model of solid tumour growth. Applied Mathematics Letters, 16(4):567 - 573, 2003.

[7] F. Cornelis, O. Saut, P. Cumsille, D. Lombardi, A. Iollo, J. Palussiere, and T. Colin. In vivo mathematical modeling of tumor growth from imaging data: Soon to come in the future? Diagnostic and Interventional Imaging, 94(6):593 - 600, 2013.

[8] A. Desmaison, C. Frongia, K. Grenier, B. Ducommun, and V. Lobjois. Mechanical stress impairs mitosis progression in multi-cellular tumor spheroids. PLOS ONE, 8(12):1-10, 122013.

[9] S. Douezan and F. Brochard-Wyart. Active diffusion-limited aggregation of cells. Soft Matter, 8:784-788, 2012.

[10] P. A. Fleming, W. S. Argraves, C. Gentile, A. Neagu, G. Forgacs, and C. J. Drake. Fusion of uniluminal vascular spheroids: a model for assembly of blood vessels. Developmental dynamics : an official publication of the American Association of Anatomists, 239(2):398-406, 022010. 
[11] E. Flenner, F. Marga, A. Neagu, I. Kosztin, and G. Forgacs. Relating biophysical properties across scales. In S. A. N. Santiago Schnell, Philip K. Maini and T. J. Newman, editors, Multiscale Modeling of Developmental Systems, volume 81 of Current Topics in Developmental Biology, pages 461 - 483. Academic Press, 2008.

[12] G. Forgacs, R. A. Foty, Y. Shafrir, and M. S. Steinberg. Viscoelastic properties of living embryonic tissues: a quantitative study. Biophysical Journal, 74(5):2227 - 2234, 1998.

[13] R. Foty, C. Pfleger, G. Forgacs, and M. Steinberg. Surface tensions of embryonic tissues predict their mutual envelopment behavior. Development, 122(5):1611-1620, 1996.

[14] R. A. Foty, G. Forgacs, C. M. Pfleger, and M. S. Steinberg. Liquid properties of embryonic tissues: Measurement of interfacial tensions. Phys. Rev. Lett., 72:2298-2301, Apr 1994.

[15] J. Frenkel. Viscous flow of crystalline bodies under the action of surface tension. $J$. Phys., 9:385, 1945.

[16] J. A. Glazier and F. m. c. Graner. Simulation of the differential adhesion driven rearrangement of biological cells. Phys. Rev. E, 47:2128-2154, Mar 1993.

[17] A. Gomes, L. Guillaume, D. R. Grimes, J. Fehrenbach, V. Lobjois, and B. Ducommun. Oxygen partial pressure is a rate-limiting parameter for cell proliferation in $3 \mathrm{~d}$ spheroids grown in physioxic culture condition. PloS one, 11(8):e0161239, 2016.

[18] B. Gompertz. On the nature of the function expressive of the law of human mortality, and on a new mode of determining the value of life contingencies. Philosophical Transactions of the Royal Society of London, 115:513-583, 1825.

[19] H. Greenspan. On the growth and stability of cell cultures and solid tumors. Journal of Theoretical Biology, 56(1):229 - 242, 1976.

[20] P. Grinfeld. Hamiltonian dynamic equations for fluid films. Studies in Applied Mathematics, 125(3):223-264, 2010.

[21] F. Hirschhaeuser, H. Menne, C. Dittfeld, J. West, W. Mueller-Klieser, and L. A. KunzSchughart. Multicellular tumor spheroids: An underestimated tool is catching up again. Journal of Biotechnology, 148(1):3 - 15, 2010. Organotypic Tissue Culture for Substance Testing.

[22] S. Hoehme and D. Drasdo. A cell-based simulation software for multi-cellular systems. Bioinformatics, 26(20):2641-2642, 2010.

[23] K. Jakab, B. Damon, F. Marga, O. Doaga, V. Mironov, I. Kosztin, R. Markwald, and G. Forgacs. Relating cell and tissue mechanics: Implications and applications. Developmental Dynamics, 237(9):2438-2449, 2008. 
[24] K. Jakab, A. Neagu, V. Mironov, R. R. Markwald, and G. Forgacs. Engineering biological structures of prescribed shape using self-assembling multicellular systems. Proceedings of the National Academy of Sciences of the United States of America, 101(9):28642869, 032004.

[25] K. Jakab, C. Norotte, F. Marga, K. Murphy, G. Vunjak-Novakovic, and G. Forgacs. Tissue engineering by self-assembly and bio-printing of living cells. Biofabrication, 2(2):022001, 2010.

[26] E. Jones, T. Oliphant, P. Peterson, et al. SciPy: Open source scientific tools for Python, 2001-. [Online; accessed jtodayi].

[27] A. Laird. Dynamics of tumour growth: comparison of growth rates and extrapolation of growth curve to one cell. Br. J. Cancer, pages 278-291, 1965.

[28] J. Laurent, C. Frongia, M. Cazales, O. Mondesert, B. Ducommun, and V. Lobjois. Multicellular tumor spheroid models to explore cell cycle checkpoints in 3D. BMC cancer, 13(1):73, 2013.

[29] T. Michel. Mathematical analysis and model calibration for tumor growth models. Theses, Université de Bordeaux, Nov. 2016.

[30] V. Mironov, R. P. Visconti, V. Kasyanov, G. Forgacs, C. J. Drake, and R. R. Markwald. Organ printing: Tissue spheroids as building blocks. Biomaterials, 30(12):2164 - 2174, 2009.

[31] J. C. Mombach, D. Robert, F. Graner, G. Gillet, G. L. Thomas, M. Idiart, and J.-P. Rieu. Rounding of aggregates of biological cells: Experiments and simulations. Physica A: Statistical Mechanics and its Applications, 352(2-4):525 - 534, 2005.

[32] O. Pokluda, C. T. Bellehumeur, and J. Vlachopoulos. Modification of Frenkel's model for sintering. Aiche Journal, 43:3253-3256, 1997.

[33] J. M. Pérez-Pomares and R. A. Foty. Tissue fusion and cell sorting in embryonic development and disease: biomedical implications. BioEssays, 28(8):809-821, 2006.

[34] E. K. Rofstad, K. Galappathi, and B. S. Mathiesen. Tumor interstitial fluid pressure-a link between tumor hypoxia, microvascular density, and lymph node metastasis. Neoplasia, pages 586-594, 2014.

[35] G. Sciumè, R. Santagiuliana, M. Ferrari, P. Decuzzi, and B. A. Schrefler. A tumor growth model with deformable ecm. Physical Biology, 11(6):065004, 2014.

[36] A. J. Shaler. Seminar on the kinetics of sintering. Metals Transactions, 185:796 - 813, 1949. 
[37] J. Spratt, D. von Fournier, J. Spratt, and E. Weber. Decelerating growth and human breast cancer. Cancer, pages 2013-2019, 1993.

[38] M. S. Steinberg. Reconstruction of tissues by dissociated cells. Science, 141(3579):401408, 1963.

[39] T. V. Stirbat, A. Mgharbel, S. Bodennec, K. Ferri, H. C. Mertani, J.-P. Rieu, and H. Delanoë-Ayari. Fine tuning of tissues' viscosity and surface tension through contractility suggests a new role for $\alpha$-catenin. PLOS ONE, 8(2):1-10, 022013.

[40] Y. Sun and Q. Wang. Modeling and simulations of multicellular aggregate self-assembly in biofabrication using kinetic Monte Carlo methods. Soft Matter, 9:2172-2186, 2013.

[41] G. L. Thomas, V. Mironov, A. Nagy-Mehez, and J. C. Mombach. Dynamics of cell aggregates fusion: Experiments and simulations. Physica A: Statistical Mechanics and its Applications, 395:247 - 254, 2014.

[42] L. Von Bertalanffy. Quantitative laws in metabolism and growth. Q. Rev. Biol, pages 217-231, 1957.

[43] A. Wessels and D. Sedmera. Developmental anatomy of the heart: a tale of mice and man. Physiological Genomics, 15(3):165-176, 2003.

[44] S. Wilson, M. Tod, A. Ouerdani, A. Emde, Y. Yarden, A. A. Berkane, S. Kassour, M. Wei, G. Freyer, B. You, E. Grenier, and B. Ribba. Modeling and predicting optimal treatment scheduling between the antiangiogenic drug sunitinib and irinotecan in preclinical settings. CPT: Pharmacometrics $\&$ Systems Pharmacology, 4(12):720-727, 2015. 